\title{
The Proverb of tama tama köl bolur in modern Turkic Languages"
}

\section{Modern Türk Dillerinde tama tama köl bolur Atasözü}

\author{
Yong-Sŏng LI ${ }^{1}$ (1)
}

*This paper was presented to the International symposium «The words of the ancestors - the source of spiritual revival» which was held on 16-17 May 2019 at the Institute of Philology and Multilingual Education of Abai Kazakh National Pedagogical University in Almaty / Kazakhstan. See http://www.kaznpu.kz/en/8147/news/ (retrieved on 24 October 2019).

${ }^{1}$ Dr., Seoul National University, Department of Asian Languages and Civilizations, Seoul, Korea

ORCID: Y.S.L. 0000-0001-8352-1212

Corresponding author/Sorumlu yazar:

Yong-Sŏng Li,

Seoul National University, Department of Asian Languages and Civilizations, Seoul 08826 Korea E-mail: yulduz77@naver.com

Submitted/Başvuru: 24.10.2019

Revision Requested/Revizyon Talebi: 29.11.2019 Last Revision Received/Son Revizyon: 30.11.2019 Accepted/Kabul: 02.12.2019

\section{Citation/Atıf:}

Li, Y. S. (2019). The Proverb of tama tama köl bolur in modern Turkic Languages. TUDED 59(2), 363-377.

https://doi.org/10.26650/TUDED2019-0103

\section{ABSTRACT}

The Turkic languages and dialects are spoken across a vast area from Pacific to the Baltic Sea, and from the Arctic Ocean to the Persian Gulf. There are several proverbs and many idioms in the Orkhon Inscriptions. Proverbs are also found in the Turkic literature of the periods after the Orkhon inscriptions. The Dīwān Luyāt at-Turk from the Karakhanid state contains many proverbs. One of them is birin birin min bolur tama tama köl bolur "One by one becomes a thousand, drop by drop becomes a pond."

This proverb is found in Ottoman Turkish as Damlaya damlaya göl olur - dušman gözü kör olur “изь капель образуется озеро, глаза врага сльпы - aus vielen Tropfen bildet sich der See, die Augen des Feindes sind blind." and Damla damla göl olur düšmen gözi kör olur "Goutte à goutte le lac se remplit; peu à peu l'oeil de l'ennemi s'affaiblit / Tropfen warden zum See; auch das Auge des Feindes mit der Zeit blind."

In the present paper we will treat this proverb and its related forms in modern Turkic languages.

Keywords: Dīwān Luүāt at-Turk, idioms, proverbs, tama tama köl bolur, Turkic languages and dialects

\section{ÖZET}

Türk dil ve lehçeleri Pasifik’ten Baltık Denizi'ne ve Kuzey Buz Denizi'nden Basra Körfezi'ne kadar uzanan geniş bir alanda konuşulur. Orhon yazıtlarında birkaç atasözü ve birçok deyim vardır. Orhon yazıtlarından sonraki dönemlere ait Türk edebiyatında da atasözleri bulunur. Karahanlı devletinden bir eser olan Dîvânü Lugâti 't-Türk birçok atasözü içermektedir. Bunlardan biri de birin birin min bolur tama tama köl bolur "Birer birer bin olur, damlaya damlaya göl olur"dur.

Bu atasözü, Osmanlı Türkçesinde Damlaya damlaya göl olur - duşman gözü kör olur ve Damla damla göl olur düşmen gözi kör olur olarak bulunur.

Bu yazıda modern Türk dillerindeki bu atasözünü ve ilgili şekillerini de ele alacağız. Anahtar Kelimeler: Dîvânü Lugâti 't-Türk, deyimler, atasözleri, tama tama köl bolur, Türk dil ve lehçeleri 


\section{EXTENDED ABSTRACT}

The Turkic languages and dialects are spoken across a vast area from Pacific to the Baltic Sea, and from the Arctic Ocean to the Persian Gulf. The modern Turkic peoples descend from an ancient grouping of tribes who, through conquest, interaction and assimilation, extended its language and elements of its culture across Eurasia. In addition to the inherited items, the Turkic languages have numerous lexical items which have been copied from non-Turkic languages.

There are several proverbs and many idioms in the Orkhon Inscriptions. Proverbs are also

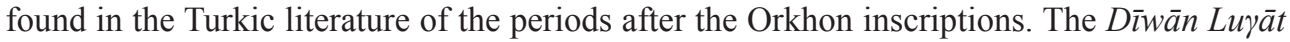
at-Turk from the Karakhanid state contains many proverbs. One of them is birin birin min bolur tama tama köl bolur "One by one becomes a thousand, drop by drop becomes a pond."

This proverb is found in Ottoman Turkish as follows:

Damlaya damlaya göl olur - dušman gözü kör olur “изъ капель образуется озеро, глаза врага сльпы - aus vielen Tropfen bildet sich der See, die Augen des Feindes sind blind.”

طمله طمله كول اولور دوشمن كوزى كور اولور Damla damla göl olur düšmen gözi kör olur “Goutte à goutte le lac se remplit; peu à peu l'oeil de l'ennemi s'affaiblit / Tropfen warden zum See; auch das Auge des Feindes mit der Zeit blind."

This proverb and its related forms in modern Turkic languages are treated in the present paper. As a result of this study, it was possible to find the following points:

1. The proverb Tama tama köl bolur is found in the same or slightly different form in many of the modern Turkic languages like Turkish, Gagauz, Azerbaijani, Turkmen, Salar, Uzbek, Uyghur, Crimean Tatar, Urum, Karaim (Crimean dial.), Karachay-Balkar, Kazakh, Karakalpak, Tatar, Bashkir, Kyrgyz, Khakas, and Chuvash.

2. The proverb Tama tama köl bolur is not found in a few languages like Kumyk, Nogay, Tuvan, and Yakut.

3. Some forms are common to the languages spoken near each other.

4. Some forms are common to the languages spoken far away from each other.

5. Some forms are found only in one language.

The proverb Tama tama köl bolur can be compared to the following proverbs of other languages:

Per. قطره قطره جمع كَردد وانكهى دريا شود Q Qtra qatra jam 'gardad wa āngahī daryā šawad "Drops accumulate and then become a river." 
Chin. 滴水成河 dīshuǐ chéng hé "Falling water drops become a river."

Rus. Капля за каплей - получается озеро; без единой капли - пустыня "Durch beständiges Tropfen entsteht ein See, ohne einen einzigen Tropfen [entsteht] eine Wüste."

Ger. Aus Tropfen wird ein großer Fluß, aus Flüssen ein See "Out of drops becomes a great river, from rivers a lake."

Mong. Дусльгх хураавал далай, Дуулсныг хураавал эрдэм “Ocean comes from collecting drops; Knowledge comes from collecting what you learn.” (Raymond 2014: 99) 


\section{Introduction}

The Turkic languages and dialects are spoken across a vast area from Pacific to the Baltic Sea, and from the Arctic Ocean to the Persian Gulf (Tekin \& Ölmez 1999: 9). The modern Turkic peoples descend from an ancient grouping of tribes who, through conquest, interaction and assimilation, extended its language and elements of its culture across Eurasia (Golden 1998: 16). In addition to the inherited items, the Turkic languages have numerous lexical items which have been copied from non-Turkic languages (Johanson 1998: 119).

There are several proverbs and many idioms in the Orkhon Inscriptions, the earliest known records of Turkic. ${ }^{1}$ Proverbs are also found in the Turkic literature of the periods after the Orkhon inscriptions. ${ }^{2}$ The Dīwān Luүāt at-Turk from the Karakhanid state contains many proverbs. ${ }^{3}$ One of them is birin birin miy bolur tama tama köl bolur "One by one becomes a thousand, drop by drop becomes a pond." (p. 602) (DLT II 333)

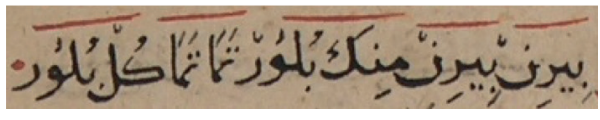

This proverb is given in Nadeljajev et al. (1969) as follows:

MID тысяча: ...; birin birin min bolur tama tama köl bolur один по одному — будет тысяча, капля по капле - будет озеро (MK III 360) (344b)

ТАМ- капать: ...; birin birin min bolur tama tama köl bolur [если взять много раз] по одному, получается тысяча, [если взять много раз] по капле (букв. капая-капая), получается озеро (QBN 201 13 ) (529b).

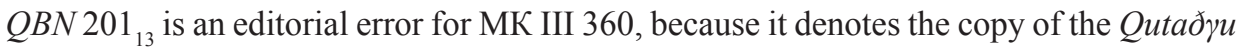
Bilig found in Namangan.

This proverb is found in Ottoman Turkish as follows:

Damlaya damlaya göl olur - dušman gözü kör olur "изь капель образуется озеро, глаза врага сльпы - aus vielen Tropfen bildet sich der See, die Augen des Feindes sind blind." (VW III 1652-1653, No. 295)

طمله طمله كول اولور دوشمن كوزى كور اولور Damla damla göl olur düšmen gözi kör olur “Goutte à goutte le lac se remplit; peu à peu l'oeil de l'ennemi s'affaiblit / Tropfen warden zum See; auch das Auge des Feindes mit der Zeit blind." (OS 96-97)

In the present paper, we will treat this proverb and its related forms in modern Turkic languages. ${ }^{4}$

For details see Tekin 1995: 19-21.

For details see Ölmez 2009: 55-63.

For details see Ölmez 2009: 58-63.

For the other proverbs see Ölmez 2009: 58-63. 


\section{2. tama tama köl bolur in modern Turkic Languages}

This proverb and its related forms appear in modern Turkic languages as follows ${ }^{5}$ :

\section{(1) Turkish}

Damlaya damlaya göl olur "Azar azar olagelen şeyler birikerek önemli bir niceliğe ulaşacağı için küçümsenmemelidir." (TS 591b), "Little by little one saves a lot" (RTED 271a), "Take care of the pennies and the pounds will take of themselves." (OTED 122a), "(Drop by drop a lake is formed.) A little at a time makes a lot." (RÇTİ 88b), "From drops to a lake." (Yurtbaş1 1993: 339a), "Durch beständiges Tropfen entsteht ein See.” (TSSR 489)

Damlyya damllya göl olur “Капля по капле - получается озеро.” (TRS 208c)

Damlaya damlaya göl olur, (aka aka sel olur) "By drops it may be a pond." (Yurtbaş1 1993: 361a)

Damlaya damlaya göl olur, aka aka sel olur "Durch beständiges Tropfen entsteht ein See, durch beständiges Fließen entsteht ein Sturzbach.” (TSSR 490)

Damlacıktan sel olur “Aus Tröpfchen wird ein Sturzbach.” (TSSR 490)

\section{(2) Gagauz}

Damnaya-damnaya göl olur “Капля по капле образует озеро.” (GRMS 118a; TSSR 489)

\section{(3) Azerbaijani}

Dama-dama göl olar, dada-dada héč "Drop by drop a lake is formed; by tasting nothing." (ADIL I 530b; Beydili 2004: 80)

Dama-dama göl olar, axa-axa sèl "Drop by drop a lake is formed; by flowing - the torrent.” (Hüseynzade 1985: 238; TSSR 490)

\section{(4) Turkmen}

Dama-dama - köl bolar, hīč dammașa čöl bolar "Капля по капле - озеро будет, нет ни капли - пустыня будет.” (TmRS 245a, 412b)

Dama-dama köl bolar, xič dammasa čöl bolar "Durch beständiges Tropfen entsteht ein See, wenn nichts tropft, entsteht eine Wüste." (TSSR 490)

Köp damjaadan köl bolar “Aus vielen Tröpfchen wird ein See.” (TSSR 490)

5 The author could find neither this proverb nor its related forms in the Turkic languages (and dialects) such as South Oghuz, Khorasan Turkic, Altay, Chulym Turkic, Yellow Uyghur, Fuyü Kyrgyz, Dolgan, and Khalaj. 
(5) Salar

Tama-tama köl volar "Drop by drop a lake is formed." (Han \& Ma 2010: 120a)

(6) Uzbek

Tåmá-tåmá köl bolur “Капля по капле образует озеро.” (URS 229b, 442a)

Tåmá-tåmá köl bolar "Drop by drop a lake is formed." (ÖTIL I 414b)

Tåmá-tåmá köl bolur, héč tåmmásá čöl bolur "Drop by drop a lake is formed; when there is not a drop - the desert is formed." (ÖTIL II 201a, 386a, 695a)

Tåma-tåma köl bolur, hèč tåmmasa čöl bolur "Drop by drop a lake is formed; when there is not a drop - the desert is formed." (TSSR 490)

(7) Uyghur

Tama-tama köl bolar "Drop by drop a lake is formed." (UyXLa 842b; UyXLb 207b; XUyL 163b; TSSR 489)

Tama-tama köl tolar "Drop by drop a lake is filled." (UyXLa 259b)

Köp tükürse köl bolur “Если все люди (букв. если многие) плюнет, образует озеро.” (UjRS 337a; UyXLa 335b; UyXLb 211a)

Köp tükürse, köl bolar "If many spit, a lake is formed." (UyXLa 817b).

\section{(8) Crimean Tatar}

Tama-tama göl olur, aqa-aqa sel olur "Drop by drop a lake is formed; by flowing the torrent is formed." (QBAT 17; TSSR 490)

Čoqluq tükürse, göl olur "If many spit, a lake is formed." (QBAT 19)

(Krymchak dial.)

Tamla-tamla göl' ol̈̈ “Капля по капле - озеро будет.” (Rebi 2004: 180)

Tamla, tamla göl’ olïr “Капля по капле - озеро будет.” (Ačkinazi 2004: 91)

(9) Urum

Tama-tama köl' boliy “Краплина по краплині - набирається озеро.” (US 272a)

Tama-tama köl' boliy/boluy “Краплина по краплині - набирається озеро.” (US 420a)

Tama-tama köl' boluy “Калюжа утворюється по краплі.” (US 110a) 
Tam-nam, tam-nam göl’ oluy “Краплина по краплині - набирається озеро.” (US 133a)

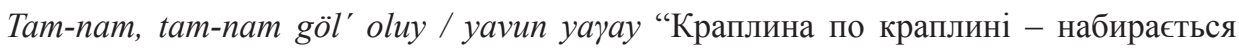
озеро / іде дощ." (US 421a)

\section{(10) Karaim (Crimean dial.)}

Damla, damla göl olur - dušman gözü kör olur "Drop by drop a lake is filled; the enemy becomes blind." (Proben II 395, No. 143)

\section{(11) Kumyk}

Tama-tama köp bolur "Drop by drop it becomes much." (Öztürk \& Uyanık 2006: 224 ; Pekacar 2006: 202)

\section{(12) Karachay-Balkar}

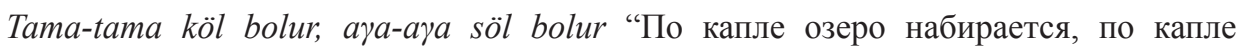
истекает." (KBRS 601a)

Tama-tama köl bolur, qona-qona el bolur "Из капель озеро образуется, из поселенцев - село." (KBRS 601a)

Köl da tama-tama boladï “По капле и озеро набирается.” (KBRS 339a), krč. “Auch der See entsteht aus einzelnen Tropfen.” (TSSR 489)

blk. Köp tamčidan köl bolur “Aus vielen Tropfen entsteht ein See.” (TSSR 490)

\section{(13) Kazakh}

Tama-tama köl bolar "Drop by drop a lake is formed." (QQS 1104b)

Tamšidan tama berse dariya bolar "If it drips from drops, a river is formed." (QQS 1106a) Tamšïdan tama berse däriya bolar "If it drips from drops, a river is formed." (QQS 306a) Tamšidan tama-tama darya bolar “Aus einzelnen Tropfen entsteht ein Fluß.” (TSSR 490) Tama berse, tamšidan dariya bolar "If it drips, a river is formed from drops." (Keykin 2014: 605)

Köp tükirse köl bolar "If many spits, a lake is formed." (QQS 1210b)

Köp tükirse köl boladï “Если все плюнут, получится море” (KzRS 398b)

Köp tükirse, köl boladï, köp birikse, yel boladï "If many spit, a lake is formed; if many [people] unite, a nation is formed." (Keykin 2014: 44) 


\section{(14) Karakalpak}

Tama-tama köl bolar, bir tambasa šöl bolar “Капля за каплей - озеро будет, ни одной капли не капнет - пустыня будет." (KkRS 616a), "Durch beständiges Tropfen entsteht ein See, wenn nichts tropft, entsteht eine Wüste.” (TSSR 490)

\section{(15) Nogay}

Köp tükirse köl bolar “Если много народу плюнет, то образуется озеро.” (NRS 178a)

Köp tükirse, köl bolar “Если много народу плюнет, то образуется озеро.” (NRS 369b)

\section{(16) Tatar}

Tamči tama-tama kül bula "Капля по капле образуется озеро.” (TtRS 336a, 514a; TSSR 489)

Il tökěrse kül bula "Если мир плюнет образуется озеро.” (TtRS 336а) (с миру по нитке - голому рубашка)

Bĕr tö̌kěrse bĕr bulïr, il tö̌kěrse kül bulïr "С миру по нитке - голому рубашка (букв. если плюнет один, то это будет одно, а если плюнет мир, то образуется озеро)" (TtRS 165a)

Il tökěrse kül bula, běr tökěrse yuk bula “Один в поле не воин (букв. если мир плюнет - озеро образуется, если один плюнет - ничего не образуется)” (TtRS 574a)

\section{(17) Bashkir}

Tamsï tamïp kül bula "По капельке образуется озеро.” (BRSa 504b), “По капельке озеро образуется." (BRSb 583b)

Tamsï tamïp kül yahay, tin yïyïlïp hum yahay “Капля за каплей образуют озеро, копейка по копейке образуют рубль.” (BRSa 504a)

\section{(18) Kyrgyz $^{6}$}

Köp tükürsö, köl bolot “Если множество плюнет, будет озеро.” (KgRS 779b; KgTTS 628b; Ibraimov 2005: 134)

6 On 20 March 2019, the author was informed about this proverb per email by Dr. Rysbek Alimov, a Kyrgyz associate professor of the Department of Turkish Language and Literature at İzmir Kâtip Çelebi University, as follows:

"Damlaya damlaya göl olur'un Kırgızcası Köp tükürsö köl bolot, yani çok kimse tükürürse, o tükürüklerden göl olur.” [The Kyrgyz form of Damlaya damlaya göl olur is Köp tükürsö köl bolot, i.e. If many people spit, it becomes lake from the spits.]

"Kırgızcada "(tamçı) tama tama köl bolot" deyişi de vardır ancak bu daha az bilinir ve daha az kullanılır." [In Kyrgyz there is also the idiom "(tamçı) tama tama köl bolot" but this is less known and less used.]

In fact, tama tama köl bolot is not found in KgRS, KgTTS, and Šambajev 1979. It seems to be the case in Kazakh, too. Tama-tama köl bolar is not found in KzRS and Keykin 2014. 
Köp tükürsö köl bolot, köl tolkusa sel bolot "Мир по слюнке плюнет - озеро будет, озеро взбушует - сель образует.” (Šambajev 1979: 206; Ibraimov 2005: 134)

Köp tükürsö - köl, köl tolkusa - sel "If many spit, a lake [is formed]; if a lake goes wild, flood [occurs].” (Ibraimov 2005: 134)

Tama - tama köl bolot "Drop by drop a lake is formed." (Ibraimov 2005: 184)

Köp tamčidan köl bolot "From many drops a lake is formed." (Ibraimov 2005: 134)

\section{(19) Khakas}

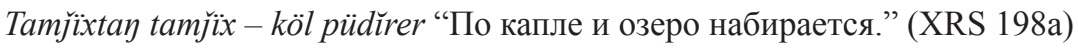

\section{(20) Tuvan}

Damdïdan dalay büder “Из капель образуется море.” (TvRSa 134a; TvRSb 146b)

\section{(21) Yakut}

Otonnōtoxxo oyōyuk tuolar “Собирать по ягодке - лукошко наполнится (соотв. капля по капле - море будет).” (JRS 274a, 280b)

\section{(22) Chuvash}

Tumlam śine tumlam - külě pulat', ulăm śine ulăm-ura pulat', pěrčé śine pěrčě-yĕtem kăšălĕ pulat' "Tropfen für Tropfen entsteht ein See, Halm für Halm entsteht ein Heuschober, Körnchen für Körnchen entsteht ein Getreidehaufen auf der Tenne.” (TSSR 489, 491)

Tumlakăn-tumlakăn tăpa tulat', pěrčěn-pěrčěn pěrne pušanat' “Tropfen für Tropfen füllt sich ein Wasser, Körnchen für Körnchen leert sich ein Korb.” (TSSR 489)

Tumlamăn-tumlamăn tăpa pulat', pěrčěn-pěrčĕn pěrne pušanat' "Tropfen für Tropfen entsteht ein Wasser, Körnchen für Körnchen leert sich ein Korb.” (TSSR 489, 491)

Tumlamran śïrma pulat' “Из капель образуется река.” (ČRSa 440a), “Aus Tropfen wird ein Bach.” (TSSR 490, 491)

Tumlamran tiněs pulat' “Aus Tropfen wird ein Meer.” (TSSR 490, 491)

Pěrer pěrčěkĕn pěrne tulat' "По зёрнышку кузов наполняется." (С̆RSa 274a), "Кузов по зёрнышку наполняется." (С̆RSb 292a), "Körnchen für Körnchen füllt sich der Korb.” (TSSR 490)

Ukśa śumne ukśa xursan, xutaś tulat' “Денежку к денежке приложишь, кисет наполнится." (С̌RSa 512b), "Wenn man Geldstück zu Geldstück legt, füllt sich der Beutel." (TSSR 490) 


\section{Conclusion}

As a result of this study, it was possible to find the following points:

1. The proverb Tama tama köl bolur is found in the same or slightly different form in many of the modern Turkic languages like Turkish, Gagauz, Azerbaijani, Turkmen, Salar, Uzbek, Uyghur, Crimean Tatar, Urum, Karaim (Crimean dial.), KarachayBalkar, Kazakh, Karakalpak, Tatar, Bashkir, Kyrgyz, Khakas, and Chuvash.

2. The proverb Tama tama köl bolur is not found in a few languages like Kumyk, Nogay, Tuvan, and Yakut.

3. Some forms are common to the languages spoken near each other.

(1) Trk. Damlaya damlaya göl olur

Gag. Damnaya-damnaya göl olur

(2) Trk. Damlaya damlaya göl olur, aka aka sel olur

Az. Dama-dama göl olar, axa-axa sèl

CTat. Tama-tama göl olur, aqa-aqa sel olur

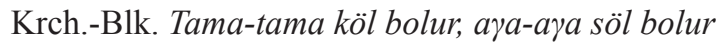

$(3)^{7}$ Tkm. Dama-dama - köl bolar, hīč dammașa čöl bolar

Uz. Tåmá-tåmá köl bolur, héč tåmmàsa čöl bolur

Kkp. Tama-tama köl bolar, bir tambasa šöl bolar

(4) Tat. Tamči tama-tama kül bula

Bash. Tamsï tamïp kül bula

(5) Uyg. Köp tükürse köl bolur / bolar

Kaz. Köp tükirse köl bolar / boladï

Kyr. Köp tükürsö, köl bolot

4. Some forms are common to the languages spoken far away from each other.

(1) Tkm. Köp damǰadan köl bolar

7 Cf. Rus. Капля за каплей - получается озеро; без единой капли - пустыня "Durch beständiges Tropfen entsteht ein See, ohne einen einzigen Tropfen [entsteht] eine Wüste.” (TSSR 490). 
Blk. Köp tamčidan köl bolur

Kyr. Köp tamčidan köl bolot

(2) Uyg. Köp tükürse köl bolur / bolar $\leftrightarrow$ CTat. Čoqluq tükürse, göl olur

Uyg. Köp tükürse köl bolur / bolar $\leftrightarrow$ Nog. Köp tükirse köl bolar

CTat. Čoqluq tükürse, göl olur $\leftrightarrow$ Kyr. Köp tükürsö, köl bolot

Nog. Köp tükirse köl bolar $\leftrightarrow$ Kyr. Köp tükürsö, köl bolot

(3) Tuv. Damdïdan dalay büder

Chuv. Tumlamran tinĕs pulat'

5. Some forms are found only in one language.

(1) Trk. Damlacıktan sel olur

(2) Az. Dama-dama göl olar, dada-dada hèč

(3) Uyg. Tama-tama köl tolar

(4) CTat. Čoqluq tükürse, göl olur

(5) Ur. Tam-nam, tam-nam yavun yayay

(6) Kar. (C) Damla, damla göl olur - dušman gözü kör olur

(7) Kmk. Tama-tama köp bolur

(8) Krch.-Blk. Tama-tama köl bolur, qona-qona el bolur

(9) Krch.-Blk. Köl da tama-tama boladï

(10) Kaz. Tamšidan tama berse dariya bolar Tama berse, tamšidan dariya bolar

(11) Kaz. Tamšïdan tama-tama darya bolar

(12) Kaz. Köp tükirse, köl boladï, köp birikse, yel boladï

(13) Tat. Il tökěrse kül bula

(14) Tat. Il tökěrse kül bula, běr tökěrse yuk bula

(15) Tat. Bĕr tökĕrse bĕr bulïr, il tö̌kĕrse kül bulïr

(16) Bash. Tamsï tamïp kül yahay, tin yïyilïp hum yahay 
(17) Kyr. Köp tükürsö köl bolot, köl tolkusa sel bolot Köp tükürsö - köl, köl tolkusa-sel

(18) Khak. Tamjüxtay tamjüx - köl püdı̌rer

(19) Tuv. Damdïdan dalay büder

(20) Yak. Otonnōtoxxo oyōyuk tuolar

(21) Chuv. Tumlam śine tumlam - külě pulat', ulăm śine ulăm-ura pulat', pěrčě śine perrče - yĕtem kăšălě pulat'

(22) Chuv. Tumlakăn-tumlakăn tăpa tulat', pěrčěn-pěrčĕn pěrne pušanat'

(23) Chuv. Tumlamăn-tumlamăn tăpa pulat', pĕrčĕn-pĕrčĕn pĕrne pušanat'

(24) Chuv. Tumlamran sïrma pulat'

(25) Chuv. Tumlamran tiněs pulat'

(26) Chuv. Pěrer pěrčěkěn pěrne tulat'

(27) Chuv. Ukśa śumne ukśa xursan, xutaś tulat'

The proverb Tama tama köl bolur can be compared to the following proverbs of other languages ${ }^{8}$ :

Per. قطره قطره جمع كردد وانگهى دريا شود Qatra qatra jam ' gardad wa āngahĩ daryā šawad "Drops accumulate and then become a river."

Chin. 滴水成河 dīshuǐ chéng hé "Falling water drops become a river."

Rus. ${ }^{9}$ Капля за каплей - получается озеро; без единой капли - пустыня "Durch beständiges Tropfen entsteht ein See, ohne einen einzigen Tropfen [entsteht] eine Wüste.” (TSSR 490)

Ger. Aus Tropfen wird ein großer Fluß, aus Flüssen ein See [“Out of drops becomes a great river, from rivers a lake.”] (TSSR 490)

Mong. ${ }^{10}$ Дусльгг хураавал далай, Дуулсньгг хураавал эрдэм “Ocean comes from collecting drops; Knowledge comes from collecting what you learn.” (Raymond, 2014: 99)

8 In connection with this, Ölmez (2009: 54) writes as follows:

"Şüphesiz bir dildeki atasözüne hemen komşu ülkenin dilinde de rastlamak mümkündür. Atasözlerinin bir kısmı çeviri yoluyla kazanılırken bir kısmı da o halkın uzun yıllara dayanan hayat tarzının ortaya koyduğu "damitılmış" sözler olabilmektedir." [Of course, the proverb in a language can be found in the language of the neighboring country. While some of the proverbs are acquired through translation, others can be "distilled" words of the people's long-term lifestyle.]

9 See also the same proverb which was on 30 March 2019 retrieved from http://posloviz.ru/category/kaplya/.

10 The author is grateful to B. Norovnyam from the Department of Mongolian Studies at Dankook University in Cheonan / Korea for her giving information about this proverb in Mongolian. 


\section{Abbreviations and Bibliography}

$\begin{array}{llll}\text { Az. } & \text { Azerbaijani } & \text { Kyr. } & \text { Kyrgyz } \\ \text { Bash. } & \text { Bashkir } & \text { Mong. } & \text { Mongolian } \\ \text { Chin. } & \text { Chinese } & \text { Nog. } & \text { Nogay } \\ \text { Chuv. } & \text { Chuvash } & \text { Per. } & \text { Persian } \\ \text { CTat. } & \text { Crimean Tatar } & \text { Rus. } & \text { Russian } \\ \text { Gag. } & \text { Gagauz } & \text { Tat. } & \text { Tatar } \\ \text { Ger. } & \text { German } & \text { Tkm. } & \text { Turkmen } \\ \text { Kar. }(\text { C) } & \text { Karaim (Crimean dialect) } & \text { Trk. } & \text { Turkish } \\ \text { Kaz. } & \text { Kazakh } & \text { Tuv. } & \text { Tuvan } \\ \text { Khak. } & \text { Khakas } & \text { Ur. } & \text { Urum } \\ \text { Kkp. } & \text { Karakalpak } & \text { Uyg. } & \text { Modern Uyghur } \\ \text { Kmk. } & \text { Kumyk } & \text { Uz. } & \text { Uzbek } \\ \text { Krch.-Blk. } & \text { Karachay-Balkar } & \text { Yak. } & \text { Yakut }\end{array}$

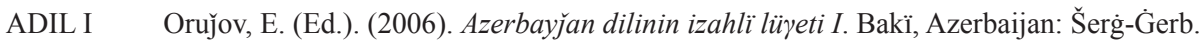

BRSa Axmerov, K. Z. et al. (1958). Baškirsko-russkij slovar'. Moskva, USSR: Gosudarstvennoje izdatel'stvo inostrannyx i nacional'nyx slovarej.

BRSb Uraksin, Z. G. (Ed.). (1996). Baškirsko-russkij slovar’. Moskva, Russia: Digora / Russkij jazyk.

ČRSa Sirotkin, M. Ja. (Ed.). (1961). Čuvašsko-russkij slovar'. Moskva, USSR: Gosudarstvennoje izdatel'stvo inostrannyx i nacional'nyx slovarej.

ČRSb Skvorcov, M. I. (Ed.). (1985). Čuvašsko-russkij slovar'. Moskva, USSR: Russkij jazyk.

DLT Dankoff, R., \& Kelly, J. (1984). Mahmūd al-Käšryarī: Compendium of the Turkic dialects (Dīwān luyāt at-Turk). Cambridge, Mass.: Office of the University Publisher, Harvard University.

GRMS Baskakov, N. A. (Ed.). (1973). Gagauzsko-russko-moldavskij slovar'. Moskva, USSR: Sovetskaja enciklopedija.

JRS Slepcov, P. A. (Ed.). (1972). Jakutsko-russkij slovar'. Moskva, USSR: Sovetskaja enciklopedija.

KBRS Tenišev, E. R., \& Sujunčev, X. I. (Eds.). (1989). Karačajevo-balkarsko-russkij slovar’. Moskva, USSR: Russkij jazyk.

KgRS Judaxin, K. K. (1965). Kirgizsko-russkij slovar'. Moskva, USSR: Sovetskaja enciklopedija.

KgTTS Abduldayev, E., \& Isayev, D. (Eds.). (1969). Kïrg̈̈z tilinin tüš̈̈ndürmö sözdügü. Frunze, USSR: Mektep.

KkRS Baskakov, N. A. (Ed.). (1958). Karakalpaksko-russkij slovar'. Moskva, USSR: Gosudarstvennoje izdatel'stvo inostrannyx i nacional'nyx slovarej.

KzRS Syzdykova, R. G., \& Xusain, K. Š. (Eds.). (2001). Kazaxsko-russkij slovar’. Almaty, Kazakhstan: Dajk-press.

NRS Baskakov, N. A. (1963). Nogajsko-russkij slovar'. Moskva, USSR: Gosudarstvennoje izdatel'stvo inostrannyx i nacional'nyx slovarej. 
OS Die K. K. Orientalische Akademie in Wien. (Ed.). (1865). Osmanische Sprichwörter. Wien, Austria: K. K. Hof- und Staatsdruckerei.

OTED Hony, H. C., \& İz, F. (1985). The Oxford Turkish-English dictionary (3 ${ }^{\text {rd }}$ ed.). Oxford, UK: Clarendon Press. [1st edition 1947]

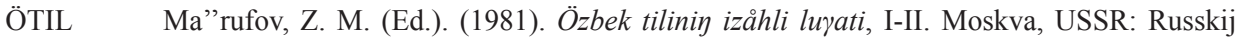
jazyk.

Proben VII Radloff, W. (1896). Proben der Volkslitteratur der nördlichen türkischen Stämme. VII. Theil. Die Mundarten der Krym. Sanktpeterburg', Russia: Kaiserliche Akademie der Wissenschaften.

QBAT Fazïl, R. (1971). Qayda birlik anda tirilik. Taškent, USSR: Ġafur Ġulam.

(2019, March 29). Retrieved from https://media.turuz.com/.../0060-Qirimtatar_atalar_ sozleri(86).pdf

QQS $\quad$ Äbikenulï, N. (2010). Qazaqša-qïtayša sözdik. Ürimži, PRC: Ulttar Baspasï.

RÇTİ Avery, C. R. et al. (Eds.). (1983). Redhouse çağdaş Türkçe-İngilizce sözlüğü. Istanbul, Turkey: Redhouse Press.

RTED Alkım, U. B. et al. (Eds.). (1988). New Redhouse English-Turkish dictionary (10 ${ }^{\text {th }}$ ed.). Istanbul, Turkey: Redhouse Press. [1st edition 1968]

TmRS Baskakov, N. A., Karryjev, B. A., \& Xamzajev, M. Ja. (Eds.). (1968). Turkmensko-russkij slovar'. Moskva, USSR: Sovetskaja enciklopedija.

TRS Mustafajev, E. M.-E., \& Starostov, L. N. (Eds.). (1977). Turecko-russkij slovar'. Moskva, USSR: Russkij jazyk.

TS Akalın, Ş. H. et al. (2011). Türkçe Sözlük. Türk Dil Kurumu Yayınları: 549. Ankara, Turkey: Türk Dil Kurumu.

TSSR Bläsing, U. (1994). Tschuwaschische Sprichwörter und sprichwörtliche Redensarten. Turkologica 20. Wiesbaden, Germany: Harrassowitz.

TtRS Abdrazakov, K. S. et al. (1966). Tatarsko-russkij slovar'. Moskva, USSR: Sovetskaja enciklopedija.

TvRSa Pal'mbax, A. A. (Ed.). (1955). Tuvinsko-russkij slovar'. Moskva, USSR: Gosudarstvennoje izdatel'stvo inostrannyx i nacional'nyx slovarej.

TvRSb Tenišev, E. R. (Ed.). (1968). Tuvinsko-russkij slovar’. Moskva, USSR: Sovetskaja enciklopedija.

UjRS Nadžip, E. N. (1968). Ujgursko-russkij slovar’. Moskva, USSR: Sovetskaja enciklopedija.

URS Borovkov, A. K. (Ed.). (1959). Uzbeksko-russkij slovar'. Moskva, USSR: Gosudarstvennoje izdatel'stvo inostrannyx i nacional'nyx slovarej.

US Garkavec', O. (2000). Urums 'kyj slovnik. Alma-Ata, Kazakhstan: Baur.

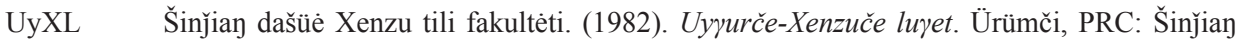
xelq nešriyati.

VW III Radloff, W. (1905). Versuch eines Wörterbuches der Türk-Dialecte III. St. Petersburg, Russia: Kaiserliche Akademie der Wissenschaften.

XRS Subrakova, O. V. (Ed.). (2006). Xakassko-russkij slovar'. Novosibirsk, Russia: Nauka. 
Ačkinazi, B. M. (2004). Qrïmčaxlarïy atalar sözï. Poslovicy i pogovorki krymčakov. Simferopol', Ukraine: Tavrija.

Ayso [阿伊草 Āyīcăo] \& Cañ C. [张进锋 Zhāng Jìnfēng] (2008). Salar ibret sözler [撒拉尔浐语 Sălāèr yànyǔ]. Xunhua Jishizhen [循化积石镇 Xúnhuà Jīshízhèn], PRC: ?.

(2019, March 31). Retrieved from https://epdf.tips/queue/salar-bret-szler.html

Baskakov, N. A., Zajączkowski, A., \& Szapszał, S. M. (Eds.). (1974). Karaimsko-russko-pol'skij slovar'. Moskva, USSR: Russkij jazyk.

Bammatov, Z. Z. (Ed.). (1969). Kumyksko-russkij slovar'. Moskva, USSR: Sovetskaja enciklopedija.

Boratav, P. N. (1964). Les Proverbs. In L. Bazin et al. (Eds.), Philologiae Turcicae fundamenta, II (pp. 67-77). Wiesbaden, Germany: Franz Steiner.

Clauson, G. (1972). An etymological dictionary of pre-thirteenth-century Turkish. Oxford, UK: Clarendon Press

Golden, P. B. (1998). The Turkic peoples: A historical sketch. In L. Johanson \& É. Á. Csató (Eds.), The Turkic languages (pp. 16-29). London, UK, \& New York, NY: Routledge.

Han J. [韩建业 Hán Jiànyè] \& Mă Ch. [马成俊 Mă Chéngjùn] (Eds.). (2010). Saweihancidian [撒维汉 词典]. Beijing, PRC: Mínzú chūbănshè [民族出版社].

Hüseynzade, E. (1985). Atalar sözü. Bakï, Azerbaijan: Yazičï.

Ibraimov, M. (2005). Kïrgïz makal-lakap, učkul sözdörü. Biškek, Kyrgyzstan: KaraBalta.

Johanson, L. (1998). .The History of Turkic. In L. Johanson \& É. Á. Csató (Eds.), The Turkic languages (pp. 81-125). London, UK \& New York, NY: Routledge.

Keykin, Ž. (2014). Qazaq maqal-mätelderiniך altïn kitabï. Almatï, Kazakhstan: Aruwna Baspasï.

(Memmedov), J. B. (2004). Atalar sözü. Bakï, Azerbaijan: Önder Nešriyyatï.

Nadeljajev, V. M. et al. (Eds.). (1969). Drevnetjurkskij slovar'. Leningrad, USSR: Nauka.

Ölmez, M. (2009). Tuva atasözlerinden seçmeler. Sibirische Studien / Sibirya incelemeleri, 4(1), 51-128.

Öztürk, R., \& Uyanık, O. (2006). Kumuk atasözleri ve deyimleri. Konya, Turkey: Çizgi Kitabevi.

Pekacar, Ç. (2006). Kumuk Türklerinin atasözleri, inceleme-metin-dizinler. Ankara, Turkey: Meyil Matbaacilik.

Peteševa, K. (2007). Altay čümdü sös. Gorno-Altaysk, Russia: Ak Čeček.

Polkanov, Ju. A. (1995). Qrïmqa[ra]ylarïy atalar-sozï / Poslovicy i pogovorki krymskix karaimov. Baxčisaraj, Ukraine: ?.

Raymond, J. (2014). Mongolian proverbs: A window into their world. Translated by Otgonjargal Oidovdorj. Eugene, Oregon: Resource Publications.

Rebi, D. (2004). Krymčakskij jazyk. Krymčaksko-russkij slovar'. Simferopol', Ukraine: DOLJA.

Roos, M., Nugteren, H., \& Zhōng, J. (1999), On some proverbs of the western and eastern Yugur languages. Turkic Languages, 3(2), 189-214.

Roos, M., Nugteren, H., \& Waibel, Z. (2006). Khakas and Shor proverbs and proverbial sayings. In M. Erdal \& I. Nevskaya (Eds.), Exploring the Eastern Frontiers of Turkic (pp. 157-192). Wiesbaden, Germany: Harrassowitz.

Stein, H. (1984). Eine türkische Sprichwortsammlung des 17. Jahrhunderts. Acta Orientalia Academiae Scientiarum Hungaricae, 38(1-2), 55-104.

Šambajev, S. (1979). Kirgizsko-russkije poslovicy, pogovorki i izrečenija. Frunze, USSR: Mektep.

Tekin, T. (1995). Orhon Yazıtları: Kül Tigin, Bilge Kağan, Tunyukuk. İstanbul, Turkey: Simurg.

Tekin, T., \& Ölmez, M. (1999). Türk dilleri - Giriş -. İstanbul, Turkey: Simurg.

Yurtbaş1, M. (1993). A dictionary of Turkish proverbs. Ankara, Turkey: Turkish Daily News.

(2019, March 30). Retrieved from http://posloviz.ru/category/kaplya/ 
